\title{
Inertial effects on thin-film wave structures with imposed surface shear on an inclined plane
}

\author{
M. Sivapuratharasu ${ }^{1}$, S. Hibberd ${ }^{1}$, M.E. Hubbard ${ }^{1}$ and H. Power ${ }^{2}$,*
}

1 School of Mathematical Sciences, University of Nottingham, Nottingham, NG7 2PB, United Kingdom

2 Faculty of Engineering, University of Nottingham, Nottingham, NG7 2PB, United Kingdom

* Auther to whom correspondence should be addressed; e-mail: henry.power@nottingham.ac.uk

\begin{abstract}
This study provides an extended approach to the mathematical simulation of thin-film flow on a flat inclined plane relevant to flows subject to high surface shear. Motivated by modelling thin-film structures within an industrial context, wave structures are investigated for flows with moderate inertial effects and small film depth aspect ratio $\varepsilon$. Approximations are made assuming a Reynolds number, $\operatorname{Re} \sim \mathcal{O}\left(\varepsilon^{-1}\right)$ and depth-averaging used to simplify the governing Navier-Stokes equations. A parallel Stokes flow is expected in the absence of any wave disturbance and a generalisation for the flow is based on a local quadratic profile. This approch provides a more general system which includes inertial effects and is solved numerically. Flow structures are compared with studies for Stokes flow in the limit of negligible inertial effects. Both two-tier and three-tier wave disturbances are used to study film profile evolution. A parametric study is provided for wave disturbances with increasing film Reynolds number. An evaluation of standing wave and transient film profiles is undertaken and identifies new profiles not previously predicted when inertial effects are neglected.
\end{abstract}

Keywords Inertial effects; Thin film; Surface shear; Wave structures; Capillarity; Numerical simulation.

\section{Introduction}

Oil films on the internal surfaces of an aero-engine bearing chamber are a primary mechanism in removing heat from the chamber as oil is continuously collected, externally cooled and recycled. In a generic bearing chamber, the oil film is typically driven by a strong shearing airflow, associated with high-speed rotating parts within the chamber. Inertial effects, relevant to high-speed applications, have been included within a recent two-dimensional film flow formulation [9]. Using this approach, leading nonlinear inertial effects are retained to analyse more general flow patterns and to provide comparison with existing thin-film asymptotic studies. Importantly the study aims to investigate the effect of inertia on some typical thin-film solutions, obtain greater insight into existing thin-film solutions and seek more general film solutions.

Lubrication theory is typically used to model general thin-film flows, based on the ratio of film thickness to a typical geometric length scale being sufficiently small and the effects of inertia negligible; i.e. taking the limit as the film Reynolds number tends to zero. However, for films subject to a high surface shear, the inertial effects at leading order may have a substantial impact in modifying high-speed film profiles, providing a general smoothing effect on the film profiles, extending the range of existing film solutions and forming possible new film profiles. Recent studies [9] within a cylindrical geometry demonstrate that modified wave structures are predicted on including film inertial effects into a film lubrication formulation.

Numerous studies involving flow on an inclined plane range from near-horizontal flows [24] to vertical falling films $[4,5,14]$. Previous studies, for example [17, 23], for shear driven flow on an inclined plane describe wave structures depending on their initial profiles as well as uniform upstream and downstream boundary conditions. Initial profiles were chosen to be of a two-tier configuration, with either the upstream or downstream film height the higher of the two. Additionally various three-tier configurations may exist in which the central section may be the highest or lowest of three heights or a successive increase or decrease in the three heights, as shown later in figures 4 and 5 .

It is common to make thin-film approximations of the Navier-Stokes equations, using the ratio $\varepsilon$ of film thickness and typical length scale of disturbances of the flow; typically the long wave equation or the integral boundary layer approach. Often used for describing falling films, the long wave equation, and other similar models, are derived using a perturbation expansion method for the stream function, determined to $\mathcal{O}(\varepsilon)$, and then substituted into the surface kinematic boundary condition. An early publication using this method by Benney [2] gives a single evolution equation for film thickness, however this has been criticised as predicting unrealistic wave profiles as the wave amplitude increases [14], as well as leading to finite-time singularities $[20,21]$. 
The integral boundary layer model takes the full Navier-Stokes equations and applies a thin-film assumption, leading to neglecting several terms. A local velocity profile, assumed parabolic by many, including $[5,14,21]$, is then substituted into this thin-film model. The Kármán-Polhausen depth-averaging technique can be applied, integrating throughout the thickness of the film to reduce the dimensionality of the system, achieving what is commonly referred to, in literature, as the Shkadov model. The Shkadov model, too, has its criticisms. It does not predict the Hopf bifurcation, necessary to foresee the formation of periodic waves on uniform thickness film flow on inclined planes [14]. Ruyer-Quil et al. [17] claim that fluid films in a moving frame of reference flowing down an inclined plane are unstable against waves when their thickness becomes larger than a specified threshold value $h_{N c}=\left(3 \mathrm{R}_{c}\right)^{\frac{1}{3}}, \mathrm{R}_{c}$ being a critical Reynolds number. The critical Reynolds number is given by Cheng and Chang [6] as $\mathrm{R}_{c}=\cot \theta$ who claim that periodic forcing applied at the inlet leads to disturbances propagating downstream whilst growing in amplitude - this happens at Reynolds numbers larger than critical [16]. An example of sinusoidal waves at the inlet which have evolved into much larger amplitude solitary pulses is given in [5]. Ruyer-Quil et al. [18] also claim that there are limitations to the Shkadov model deriving from the lack of freedom in the description of the hydrodynamic fields, due to the nature of the depth-averaging technique. Notably, the simplification has the benefit of reduced computational cost on reducing the dimensionality of the Navier-Stokes equation.

Furthermore, thin-film flows having free surface shock structures are known to form capillary ripples at the front of the shock. In some cases, breakdown of the numerical results can occur in the simulation of Shkadov models due to over-prediction of capillary wave amplitudes, resulting in very thin film thickness. The accuracy of the Shkadov model in predicting capillary ripples is shown to decrease with increasing Reynolds number (see figures 4 and 5 of [18]), with larger than actual capillary waves being predicted. Indeed, an essential assumption of this model concerns the number of degrees of freedom of polynomials used to approximate the crosswise distribution of the streamwise velocity. A study by Malamataris et al. [13] observes that a self-similar parabolic profile provides a good approximation of the velocity field in regions where deviations are small, but also concludes that there is a change of behaviour around the region in front of a wave hump. Computed velocity profiles with backflow are depicted in figure 7 of [13], with potential inflexion points which could indicate that a cubic profile may be a more suitable approximation in this region. This is in agreement with work by Samanta et al. [20], who conclude that in a nonlinear regime, the backflow phenomenon is shown to be intensified by a no-slip condition enforced between the fluid and the plane. Backflow in the capillary region is investigated experimentally by Dietze et al. [7]. It should also be noted that Malamataris et al. comment on a parabolic profile, which is the exact solution of uniform film flow, as making the problem analytically tractable. Studies by Prokopiou et al. [16] and Yu et al. [25] extend the Shkadov model in order to allow for higher order description of film profiles relevant at higher Reynolds numbers. Referred to as the second-order boundary layer model, this retains terms of $\mathcal{O}\left(\varepsilon^{2}\right)$, and hence the modified model includes additional viscous terms, tangential and normal stress conditions and pressure variations across the film.

An investigation of travelling wave solutions on an inclined plane by Benilov [1] describes a depth-averaged approach based on the Stokes equations, i.e. neglecting inertial effects. It is suggested in this study that the model can be extended with a term accounting for surface tension.

The above mentioned literature predominantly investigate gravity driven film flow. Several other studies take similar approaches but with the imposition of surface shear. Kay et al. [9] study two-dimensional thinfilm rimming flow within a fixed cylindrical geometry subject to surface shear. Extending previous leadingorder thin-film models, inertial effects are included relevant to very high surface shear and correspondingly high-speed film flow applications. Using a hydraulic model approach enabled leading non-linear inertial effects to be retained. Kay et al. use a local quadratic velocity profile for its consistency with lubrication theory, but also extended to a cubic velocity profile, where the extra profile parameter permits modelling of wall roughness effects. Important to this study, the effects of inertia on some typical rimming-flow solutions provide new and greater insight into existing film solutions.

Samanta [19] investigates the mechanism of instability in shear imposed flow on an inclined plane. The geometry of the problem studied is very similar to the model we propose but with disturbances generated at the inlet which evolve into periodic travelling waves. There are also significant differences in the order of scaling used - the dimensionless governing equations include diffusive terms which, as will later be shown, are disregarded in our formulation due to the nature of the thin-film approximations. Another important comparison is the magnitude of the Reynolds number which differs from what we term the reduced Reynolds number by $\varepsilon$. The avenue of exploration in [19] is predominantly concerning the linear instability threshold 
and recovering the critical Reynolds and Froude numbers for the shear-imposed falling film. Although we will not be conducting any formal stability analysis, section 5 contains some discussion about the evolution of perturbations in order to characterise waves structures.

A numerical study based on the Stokes equations using a boundary element approach was given by Shuaib et al. [23] to determine the different patterns of possible wave structures which can be observed on a thin film flowing on an inclined plane when subject to a constant surface shear, and categorises conditions for different types of film profiles. Studies by Bertozzi et al. [3] proposed that there are several different types of shock waves that connect films of different heights on an inclined plane when the flow is driven by surface shear and gravity. Solutions with more than one type of shock wave were also reported, dependent on the initial configuration of the flow. Bertozzi reports three different types of wave profiles, namely compressive shocks, undercompressive shocks and rarefaction waves. In order to obtain the classification of the type of wave, a shock speed $S$, given by the Rankine-Hugoniot condition

$$
S=\frac{Q_{-}-Q_{+}}{H_{-}-H_{+}},
$$

is compared with the velocity $F_{ \pm}$of propagation of small disturbances. In (1.1) $H_{ \pm}$and $Q_{ \pm}$are the fluid depth and flux respectively, with + and - referring to values either side of the shock. A compressive shock satisfies the Lax shock inequalities $F_{+} \leq S \leq F_{-}$, assuming travel from left to right; a non-classical shock satisfies the inequalities $S \geq F_{-}$and $S \geq F_{+}$, and is also known as an undercompressive shock. The third type of structure is a rarefaction wave - a smooth, continuous profile gradually joining $H_{-}$and $H_{+}$.

This study investigates the importance of inertia on possible two-dimensional film profiles on an inclined plane under constant surface shear. The geometry is illustrated in figure 1, where uniform flow solutions can exist corresponding to three possible conjugate height and wave solutions. Validation of the modelling and numerical calculations will be sought from comparisons with previously published literature, including Shuaib [23] and Bertozzi [3], in the limit of negligible inertial terms. To investigate leading order inertial effects on the film profile, especially in regions where the film profile changes rapidly, modelling will be restricted to at most moderate Reynolds numbers (see Kay et al. [9]). Depth-averaging as used by Benilov, Bertozzi, and Kay [1, 3, 9] and many other authors, is used to reduce the governing equations to fewer dependent variables and leads to Shkadov-type integral boundary layer model upon adoption of an assumed velocity profile. A pair of coupled partial differential equations for film thickness and local film flux result.

It is noted that there exists some criticisms of an integral boundary layer approach. Some of these problems arise when investigating surface waves periodically forced at the inlet, such as in [5]. The other issue raised with the Shkadov model is that capillary ripples at the base of the main shock are exaggerated and can in some cases cause the numerical simulation to exhibit a singularity. The present work investigates moving shock structures defined between the thickness of different uniform flow conditions. However, as we will show later in section 5 , the inclusion of surface shear decreases the capillary wave amplitudes while increasing their wavelength, allowing the prediction of wave profiles with shock structures and concurrent front capillary ripples at moderate Reynolds number with the use of Shkadov depth-averaged models.

For flow down an inclined plane, a constant-height exact basic flow configuration with quadratic longitudinal velocity profile, known as the Nusselt solution, is considered far away from any shock structure. For a specified steady value of flux, this can provide up to three physically valid conjugate Nusselt heights. Additional solutions with transitions from the conjugate states may be able to coexist to form a piecewise steady film profile. Shuaib has investigated both travelling wave and transient film profiles whilst neglecting the effects of inertia. A major aim of this study is to extend current studies through investigating the impact of inertial terms present in the Navier-Stokes equations, on the thin-film shock structures formed on an inclined plane.

In section 2 we look at the formulation of the mathematical model, before progressing to evaluate this model in the context of a uniform Stokes flow in section 3. Application of thin film approximations are used and analysis of the resulting depth-averaged approach is provided in section 4 along with a brief summary of the numerical solver. Numerical results corresponding to enhanced understanding of existing and new features are included section 5. Concluding remarks in section 6 provide an evaluation of limiting solutions for moderate Reynolds number film flow in comparison with Stokes flow solutions. 


\section{Model formulation}

A Cartesian coordinate system is used to describe the flow down a flat, inclined plane with the velocity vector $\overline{\mathbf{u}}=(\bar{u}, \bar{v})$, where $\bar{u}$ is the component of the velocity in the $\bar{x}$-direction and $\bar{v}$ is the component in the $\bar{y}$-direction. The angle of inclination, $\bar{\theta}$, is measured in the anticlockwise direction from the horizontal, and so the angle depicted in figure 1 would be negative. The flow is subject to a constant surface shear $\bar{\tau}$ opposing gravity and a pressure $\overline{p_{a}}$ at the free surface with typical wave speed $U_{0}$. For this study, we choose a transformation to a moving coordinate system with the plane moving with fixed speed $U_{0}$ in the negative $\bar{x}$-direction, as shown in figure 1 .

The film is assumed to be incompressible, with constant density $\rho$ and constant viscosity $\mu$. Gravity is acting with components $\overline{\mathbf{g}}=(g \sin \bar{\theta},-g \cos \bar{\theta})$. The unit normal vector $\overline{\mathbf{n}}$ and the unit tangential vector $\overline{\mathbf{t}}$ to the free surface are

$$
\overline{\mathbf{n}}=\left(1+\bar{h}_{\bar{x}}^{2}\right)^{-\frac{1}{2}}\left(-\bar{h}_{\bar{x}}, 1\right), \quad \overline{\mathbf{t}}=\left(1+\bar{h}_{\bar{x}}^{2}\right)^{-\frac{1}{2}}\left(1, \bar{h}_{\bar{x}}\right) .
$$

The incompressible Navier-Stokes equations are taken as the governing equations - conservation of mass is described by

$$
\bar{u}_{\bar{x}}+\bar{v}_{\bar{y}}=0 .
$$

and, taking $\nu=\frac{\mu}{\rho}$, the conservation of momentum is given by

$$
\begin{aligned}
& \bar{u}_{\bar{t}}+\bar{u} \bar{u}_{\bar{x}}+\bar{v} \bar{u}_{\bar{y}}=-\frac{1}{\rho} \bar{p}_{\bar{x}}+\nu\left(\bar{u}_{\bar{x} \bar{x}}+\bar{u}_{\bar{y} \bar{y}}\right)+g \sin \bar{\theta} \\
& \bar{v}_{\bar{t}}+\bar{u} \bar{v}_{\bar{x}}+\bar{v} \bar{v}_{\bar{y}}=-\frac{1}{\rho} \bar{p}_{\bar{y}}+\nu\left(\bar{v}_{\bar{x} \bar{x}}+\bar{v}_{\bar{y} \bar{y}}\right)-g \cos \bar{\theta}
\end{aligned}
$$

No-slip and no-penetration boundary conditions are imposed at the wall giving

$$
\bar{u}=-U_{0} \quad \text { and } \quad \bar{v}=0 \quad \text { on } \quad \bar{y}=0
$$

and the kinematic condition on the free surface gives

$$
\bar{h}_{\bar{t}}+\bar{u} \bar{h}_{\bar{x}}=\bar{v} \quad \text { on } \quad \bar{y}=\bar{h}(\bar{x}, \bar{t})
$$

Taking the surface of the film to have surface tension $\sigma$, a balance of forces on the interface between liquid and gas, when written in component form [17], leads to the normal stress boundary condition,

$$
\sigma \bar{h}_{\bar{x} \bar{x}}\left(1+\bar{h}_{\bar{x}}^{2}\right)^{-\frac{3}{2}}+2 \mu\left(1+\bar{h}_{\bar{x}}\right)^{-2}\left(\bar{h}_{\bar{x}}\left(\bar{u}_{\bar{y}}+\bar{v}_{\bar{x}}\right)-\bar{h}_{\bar{x}}^{2} \bar{u}_{\bar{x}}-\bar{v}_{\bar{y}}\right)+\bar{p}-\bar{p}_{a}=0 \quad \text { on } \quad \bar{y}=\bar{h}
$$

and the tangential stress condition,

$$
\bar{\tau}=2 \mu \bar{h}_{\bar{x}}\left(\bar{v}_{\bar{y}}-\bar{u}_{\bar{x}}\right)+\mu\left(1-\bar{h}_{\bar{x}}^{2}\right)\left(\bar{u}_{\bar{y}}+\bar{v}_{\bar{x}}\right) \quad \text { on } \quad \bar{y}=\bar{h} .
$$

The model is non-dimensionalised using the following choice of scalings:

$$
\begin{aligned}
\bar{h}=h_{0} h, \bar{x}=\frac{h_{0}}{\varepsilon} x, \bar{y}=h_{0} y, \bar{u}=U_{0} u, \bar{v}=\varepsilon U_{0} v, \bar{p}=\frac{\mu U_{0}}{\varepsilon h_{0}} p, \overline{p_{a}}=\frac{\mu U_{0}}{\varepsilon h_{0}} p_{a}, \\
\bar{t}=\frac{h_{0}}{\varepsilon U_{0}} t, \bar{\theta}=\theta \quad \text { and } \quad \bar{\tau}=\frac{\mu U_{0}}{h_{0}} \tau .
\end{aligned}
$$

$U_{0}$ is the speed of the wall, $h_{0}$ is a typical height of the film and $\frac{h_{0}}{\varepsilon}$ is an $\bar{x}$-lengthscale, where $\varepsilon \ll 1$ is the ratio of vertical film thickness to the horizontal lengthscales associated with a wave disturbance. This choice of non-dimensionalisation is typical of a thin-film model.

The Navier-Stokes equations (2.2)-(2.4) in the non-dimensional variables are

$$
\begin{aligned}
u_{x}+v_{y} & =0 \\
\varepsilon \operatorname{Re}\left(u_{t}+u u_{x}+v u_{y}\right) & =-p_{x}+\varepsilon^{2} u_{x x}+u_{y y}+\lambda \sin \theta \\
\varepsilon^{3} \operatorname{Re}\left(v_{t}+u v_{x}+v v_{y}\right) & =-p_{y}+\varepsilon^{4} v_{x x}+\varepsilon^{2} v_{y y}-\varepsilon \lambda \cos \theta
\end{aligned}
$$


where the film Reynolds number $\operatorname{Re}=\frac{U_{0} h_{0}}{\nu}$ is the ratio of inertial to viscous forces and $\lambda=\frac{\rho g h_{0}^{2}}{\mu U_{0}}=\frac{\mathrm{Re}}{\mathrm{Fr}}$. The Froude number, $\mathrm{Fr}=\frac{U_{0}^{2}}{g h_{0}}$, is the ratio of inertial to gravitational forces. The non-dimensional form of the kinematic boundary condition $(2.6)$ is

$$
h_{t}+u h_{x}=v \quad \text { on } \quad y=h
$$

and the no-slip and no-penetration conditions (2.5) become

$$
u=-1 \text { and } v=0 \text { on } y=0 \text {. }
$$

The normal stress boundary condition (2.7) gives

$$
\frac{\varepsilon^{3}}{\mathrm{Ca}} h_{x x}\left(1+\varepsilon^{2} h_{x}{ }^{2}\right)^{-\frac{3}{2}}+2\left(1+\varepsilon^{2} h_{x}\right)^{-2}\left(\varepsilon^{2} h_{x}\left(u_{y}+\varepsilon^{2} v_{x}\right)-\varepsilon^{4} h_{x}^{2} u_{x}-\varepsilon^{2} v_{y}\right)+p-p_{a}=0 \quad \text { on } \quad y=h .
$$

The capillary number, $\mathrm{Ca}=\frac{\mu U_{0}}{\sigma}$, relates the effects of viscosity to surface tension across the interface between a liquid and a gas, although in various other formulations [14, 21], the Weber and Kapitza numbers have been included as a similar comparison between surface tension and other fluid forces. Gjevik [8] takes the Weber number to be of $\mathcal{O}\left(\varepsilon^{-2}\right)$, corresponding to a choice of Capillary number in our formulation of $\mathcal{O}\left(\varepsilon^{-2}\right)$ - resulting in an overall surface tension term of $\mathcal{O}(\varepsilon)$. The tangential stress condition (2.8) becomes

$$
\tau=2 \varepsilon^{2} h_{x}\left(v_{y}-u_{x}\right)+\left(1-\varepsilon^{2} h_{x}^{2}\right)\left(u_{y}+\varepsilon^{2} v_{x}\right) \quad \text { on } \quad y=h .
$$

\section{Uniform flow down an inclined plane}

In the context of Stokes flow, we take the limit $\mathrm{Re} \ll 1$ and the governing equations become

$$
\begin{aligned}
u_{x}+v_{y} & =0, \\
-p_{x}+\varepsilon^{2} u_{x x}+u_{y y}+\lambda \sin \theta & =0 \\
-p_{y}+\varepsilon^{4} v_{x x}+\varepsilon^{2} v_{y y}-\varepsilon \lambda \cos \theta & =0 .
\end{aligned}
$$

Thin-film flow studies evaluate equations (3.1)-(3.3) in the context of lubrication theory, with $\varepsilon \ll 1$. We consider unidirectional uniform flow by taking the component of the velocity perpendicular to the plane $v=0$ at leading order. Thus, assuming no variation in the $x$-coordinate direction, (3.2) is reduced to

$$
u_{y y}+\lambda \sin \theta=0 .
$$

Together with the no-slip boundary condition (2.14) on the lower wall and the surface shear condition (2.16) on the free surface, which becomes

$$
u_{y}=\tau \quad \text { on } \quad y=h
$$

when $\varepsilon \ll 1$, this yields a quadratic velocity profile for the component of film velocity parallel to the inclined plane

$$
u=\tau y+\lambda\left(h y-\frac{1}{2} y^{2}\right) \sin \theta-1
$$

The film flux is calculated by integrating the velocity profile, $q=\int_{0}^{h} u \mathrm{~d} y$, giving

$$
q=\frac{\lambda h^{3}}{3} \sin \theta+\frac{\tau h^{2}}{2}-h
$$

For a fixed flux and for specified values of $\lambda, \theta$ and $\tau$, the cubic polynomial (3.7) yields up to three possible conjugate heights for a uniform profile. In order for three distinct values of $h$ satisfying the same $q$, the range of values which surface shear $\tau$ may take is limited, as can be seen in figure 2. The curves for $\tau=1.8320$ and $\tau=2.6171$ (to 4 d.p.) represent limiting case scenarios which result in two distinct heights for a given flux, beyond which there is only a single height possible. 


\section{Thin-film calculation at moderate Reynolds numbers}

\subsection{Approximations at $\operatorname{Re} \sim \mathcal{O}\left(\varepsilon^{-1}\right)$}

Thin films subject to strong surface shear may experience moderate inertial effects at leading order on taking the following formal approximations:

$$
\varepsilon \ll 1, \quad \operatorname{Re} \sim \mathcal{O}\left(\varepsilon^{-1}\right) \quad \text { and } \quad \lambda \sim \mathcal{O}(1) .
$$

These lead to moderate values of Reynolds number that can be compared to classical lubrication theory [23], where $\varepsilon \ll 1$ and $\operatorname{Re} \rightarrow 0$. The Navier-Stokes equations $(2.10)-(2.12)$, to $\mathcal{O}(\varepsilon)$, are

$$
\begin{aligned}
u_{x}+v_{y} & =0, \\
\operatorname{Re}^{*}\left(u_{t}+u u_{x}+v u_{y}\right) & =-p_{x}+u_{y y}+\lambda \sin \theta, \\
p_{y}+\varepsilon \lambda \cos \theta & =0
\end{aligned}
$$

where $\mathrm{Re}^{*}=\varepsilon$ Re is the reduced Reynolds number. The no-slip and no-penetration conditions remain.

In considering the normal stress boundary condition (2.15), we retain the surface tension term involving $h_{x x}$ although it is negligible in the majority of the flow as it can become comparable to the other terms in regions of any shock transitions between conjugate height solutions and therefore may have a dominant local effect on the flow. This approach is consistent also with other thin-film studies $[8,9,14]$ and the normal stress condition (2.15) is approximated as

$$
\frac{\varepsilon^{3}}{\mathrm{Ca}} h_{x x}+p-p_{a}=0 \quad \text { on } \quad y=h .
$$

The tangential stress boundary condition (2.16) under the above approximation is

$$
\tau=u_{y} \quad \text { on } \quad y=h .
$$

\subsection{Integral boundary layer model}

Depth-averaging is used to reduce the system of equations by integrating (4.2)-(4.4) from the wall at $y=0$ through to the surface of the film at $y=h$. Kay [9] uses a similar technique to remove radial dependence in a film flow within a polar coordinate system.

Integrating the continuity equation (4.2) and incorporating the no-slip, no-penetration and kinematic boundary conditions gives

$$
h_{t}+q_{x}=0,
$$

where $q$ is the dimensionless volume flux. Integrating equation (4.4) and applying the normal stress boundary condition (4.5) gives the pressure within the film as

$$
p=p_{a}+\varepsilon \lambda(h-y) \cos \theta-\frac{\varepsilon^{3}}{\mathrm{Ca}} h_{x x}
$$

Depth-averaging of equation (4.3), after some algebraic manipulation, gives

$$
\operatorname{Re}^{*}\left(q_{t}+\frac{\partial}{\partial x} \int_{0}^{h} u^{2} \mathrm{~d} y\right)=\lambda h \sin \theta+\tau-\left.u_{y}\right|_{y=0}-\varepsilon \lambda h h_{x} \cos \theta+\frac{\varepsilon^{3}}{\mathrm{Ca}} h h_{x x x} .
$$

We now assume a quadratic form of the film profile similar to Nguyen and Balakotaiah [14], who use a stream function of the form $\psi(x, y, t)=A(x, t) y^{2}+B(x, t) y^{3}$. A quadratic general velocity profile at $\mathcal{O}(1)$ is written as

$$
u=a_{0}+a_{1} y+a_{2} y^{2},
$$

where the coefficients $a_{0}, a_{1}$ and $a_{2}$, which may vary with position in the plane $x$, can be calculated using the surface shear condition on $y=h$ and the no-slip condition on $y=0$, with volume flux $q$ and shear $\tau$. Application of these conditions gives expressions for the profile coefficients as

$$
a_{0}=-1, \quad a_{1}=\frac{3}{h}-\frac{\tau}{2}+\frac{3 q}{h^{2}} \quad \text { and } \quad a_{2}=-\frac{3}{2 h^{2}}+\frac{3 \tau}{4 h}-\frac{3 q}{2 h^{3}} .
$$


The profile (4.10) is used to evaluate the integral term in equation (4.9). By substitution and simplification of the associated algebra, (4.9) can be written as

$$
\operatorname{Re}^{*}\left(q_{t}+I_{1} h_{x}+I_{2} q_{x}\right)=\lambda h \sin \theta-\frac{3}{h}+\frac{3 \tau}{2}-\frac{3 q}{h^{2}}-\varepsilon \lambda h \cos \theta h_{x}+\frac{\varepsilon^{3}}{\mathrm{Ca}} h h_{x x x} .
$$

$I_{1}$ and $I_{2}$ are functions of $h, q$ and $\tau$, given by

$$
I_{1}=\frac{4 h^{3} \tau+h^{4} \tau^{2}-48 q^{2}+2 q h^{2} \tau+8 h^{2}}{40 h^{2}} \quad \text { and } \quad I_{2}=\frac{8 h+h^{2} \tau+48 q}{20 h} .
$$

Equations (4.7) and (4.12) compare directly with the coupled partial differential equations of the Shkadov model [22]. As commented in the introduction, the system of equations (4.7) and (4.12) is used here for the numerical simulation of moving shock structures on thin films that are subject to imposed exterior surface shear. Although differences between the present formulation and the Shkadov model are slight, their implications on the numerical simulations reported here are significant. A key difference is that the Shkadov model does not incorporate surface shear $(\tau=0)$. The inclusion of surface shear, as shown later in section 5.1 , leads to stabilisation of the capillary ripples and allows for numerical simulations at higher Reynolds numbers.

\subsection{Film evolution equations}

Following from the previous section, film height and film flux are given from solving

$$
\operatorname{Re}^{*}\left(q_{t}+I_{1} h_{x}-I_{2} h_{t}\right)-\lambda h \sin \theta+\frac{3}{h}-\frac{3 \tau}{2}+\frac{3 q}{h^{2}}+\varepsilon \lambda h \cos \theta h_{x}-\frac{\varepsilon^{3}}{\mathrm{Ca}} h h_{x x x}=0
$$

and

$$
h_{t}+q_{x}=0 .
$$

These are subject to general constant far-field boundary conditions

$$
h=h_{l}, q=q_{l} \quad \text { as } \quad x \rightarrow-\infty
$$

and

$$
h=h_{r}, q=q_{r} \quad \text { as } \quad x \rightarrow \infty .
$$

\subsection{Numerical computation}

The governing equations (4.14)-(4.15) and associated boundary conditions are solved numerically on a computational domain $0<x<L$, discretised by $n-1$ interior nodes $x_{i}$, for $i=1 \ldots n-1$; domain selection is sufficient for flow at $x_{0}$ and $x_{n}$ to be assumed uniform. Values of $q$ and $h$ are given at $x_{0}$ and $x_{n}$.

A finite difference approach is used to calculate the approximate values of the spatial derivatives at each of the interior points. A linear upwind differencing scheme (LUDS) [15] provides an approximation of the first derivative of film height, $h_{x}$, and for the third derivative $h_{x x x}$ we use a second-order central difference.

It is assumed that the film profile is sufficiently level at the boundaries of the computation domain. We employ an implicit first-order Euler method [11] to approximate time variation in both the momentum and continuity equations. This yields a system of $2 n-2$ equations for values of grid variables $h_{1}, \ldots, h_{n-1}$ and $q_{1}, \ldots, q_{n-1}$. An iterative solver based on Newton's method is used to find a solution at each time-step. As with other Newton method based iterative procedures, we anticipate quadratic convergence given a suitable initial guess. The initial profile for the first time-step is chosen as a smoothed function dependent on values of $h$ given by the cubic equation (3.7), and subsequent initial profiles for Newton's method are taken as the converged solution from the previous time-step.

\section{$5 \quad$ Film profile solutions}

Solutions obtained from the inertial model developed in this study may be validated against the non-inertial limiting case film profiles obtained in previous literature. Shuaib [23] has investigated a similar problem in the context of Stokes flow. Taking Re $=0$ gives an expression for flux,

$$
q=\frac{h^{2}}{3}\left(\lambda h \sin \theta-\frac{3}{h}+\frac{3 \tau}{2}-\varepsilon \lambda h \cos \theta \frac{\partial h}{\partial x}+\frac{\varepsilon^{3}}{\mathrm{Ca}} h \frac{\partial^{3} h}{\partial x^{3}}\right)
$$


and insertion of (5.1) into (4.15) yields, in non-dimensional form,

$$
\frac{\partial h}{\partial t}+\frac{\partial}{\partial x}\left(\frac{1}{2} \tau h^{2}+\frac{1}{3} \lambda h^{3} \sin \theta\right)=-\frac{\varepsilon^{3}}{3 \mathrm{Ca}} \frac{\partial}{\partial x}\left(h^{3} \frac{\partial^{3} h}{\partial x^{3}}\right)+\frac{\partial}{\partial x}\left(\frac{1}{3} \varepsilon \lambda h^{3} \cos \theta \frac{\partial h}{\partial x}\right)+\frac{\partial h}{\partial x} .
$$

Equation (5.2) is comparable to the evolution equation for the film thickness $h$ in [23] given that the appropriate parameter values are chosen. Hence, solutions obtained by Shuaib should correspond to setting the reduced Reynolds number to be zero and choosing our parameters as

$$
\lambda=1, \mathrm{Ca}=1 \times 10^{-4}, \tau=2.14914, q=-0.2068 \text { and } \varepsilon=1 \times 10^{-2} .
$$

A reference velocity corresponding to the dimensional velocity of the wall is calculated by inserting the dimensional equivalent of the cubic expression for flux within a uniform flow (3.7) into the RankineHugoniot condition (1.1). The flux in (1.1) corresponds to a stationary reference system, and so we make the substitution $\hat{q}=q+h$, where $\hat{q}$ and $q$ are in the fluxes in the stationary and moving reference frames respectively. With the appropriate algebraic manipulation, we obtain

$$
U_{0}=\left(\frac{\rho g \sin \theta h_{0}^{2}}{3 \mu}\right)\left(\frac{1-b^{3}}{1-b}\right)+\left(\frac{\tau h_{0}}{2 \mu}\right)\left(\frac{1-b^{2}}{1-b}\right),
$$

where $b$ is the ratio of the film heights at the upper and lower ends of the plane respectively. The dimensional reference velocity may be used to obtain more information about speed and direction of propagation of fronts to aid with classification of the different types of wave features. Within a moving frame of reference, we consider the motion of perturbations relative to the front. Perturbations on either side of a Lax shock travel towards the compressive front and impinge upon it (figure 3a); perturbations either side of an undercompressive front appear to move through the feature with both perturbations propagating in the same direction (figure $3 \mathrm{~b}$ ). A rarefaction wave appears to be spreading out, with perturbations either side moving away from the wave feature (figure 3c). Figure 3 depicts upstream facing structures with flow from right to left in a moving frame of reference - downstream facing structures are classified as reverse compressive or reverse undercompressive.

For a given slope inclination, conjugate values of $h$ are calculated by specifying flux $q$, surface shear, $\tau$, and gravity parameter, $\lambda$. Solving the cubic equation (3.7) yields $h_{1}, h_{2}$ and $h_{3}$ (see table 1 ), all of which can theoretically coexist for the values of $\tau$ chosen.

Wave structures are instigated from defining an initial film profile and we investigate how standing waves and transient film profiles evolve. Two-tier initial profiles are illustrated in figures $4 \mathrm{a}$ and $4 \mathrm{~b}$ that involve choosing two of the conjugate heights $h_{1}<h_{2}<h_{3}$ for given values of $\theta$ and $\tau$. Figures 5a to 5f illustrate three-tier initial profiles involving all of the allowable conjugate heights. For ease of notation, we label the height of the initial film profile on the left hand side of the domain $h_{l}$, height on the right hand side $h_{r}$ and, if applicable, the height of the middle tier $h_{m}$.

Results are included for $\theta=-45$, which are typical of profiles obtained at most slope inclination angles and for $\theta=-80$, where exceptions were observed. Features within profiles are classified as: compressive (C), reverse compressive (RC), undercompressive (UC), reverse undercompressive (RUC) or a rarefaction wave $(\mathrm{Rw})$.

Solutions reported in this work that appear as stationary are standing shocks moving up the plane with the reference velocity (5.4) in the corresponding fixed physical coordinate system, while those that appear as travelling fronts are moving faster or slower than the reference velocity. In all the cases reported here the imposed surface shear overcomes the gravitational force and the resulting fronts always move upwardly in the plane.

\subsection{Smoothing effects of inertia}

An initial film profile of the form in figure 4a develops into a compressive wave-front under initial conditions where $h_{l}=h_{2}$ and $h_{r}=h_{1}$, as shown in figure 6 . Results are comparable to the non-inertial profiles reported by Levy and Shearer [12], and Shuaib [23]. Increasing the Reynolds number results, as expected from increasing film inertial effects, in a smoothing of the film profile at the top of the shock. In addition, we find the formation of very short wavelength capillary ripples at its base, as shown in figure 6c. These capillary ripples are mesh-independent, indicating this is a model feature. They are also present in profiles obtained 
in other numerical studies which include the effects of inertia [19], as well as observed in experimental work by Kofman et al. [10]. Further investigations were carried out by varying the surface shear. As previously noted for figure 2, there is a limitation to the range of values $\tau$ is able to take in order to support three unique film depths. Expanded plots of the capillary ripples for three different values of $\tau$ are given in figure 7. It can be seen that with increasing surface shear, the wavelength of the capillary ripples also increases, while the amplitude decreases. This aids with numerical stability of the solution and hence, we were able to achieve solutions for the full range of values for which our approximation $\operatorname{Re} \sim \mathcal{O}\left(\frac{1}{\varepsilon}\right)$ is valid without any finite-time singularities.

Wave structures are typically classified as either compressive, undercompressive or a rarefaction wave using the direction of travel of perturbations. An example of this is given in figure $6 \mathrm{~b}$, which contains the film profile in figure 6 a together with finite-amplitude perturbations on either side of the shock front. It can be seen the the perturbations either side of the front are travelling towards the front in the moving frame of reference, and therefore this is classified as a compressive shock. Another point to note from figure $6 \mathrm{~b}$ is that the perturbations decay in amplitude as they travel towards the shock front without any numerical instability. This was found in all other cases investigated and reported here, suggesting that film profiles are stable to finite-amplitude perturbations (nonlinear stability) and that imposing surface shear on the integral boundary layer model makes it less susceptible to singularities and numerical instabilities than reported of the Shkadov model in previous literature.

Figure 8 , with boundary conditions as in figure 6 , but an intermediate tier in the initial profile at $h_{m}=h_{3}$, shows the time development of a compressive wave front at $\operatorname{Re}^{*}=0$. The initial peak in figure 8 a decreases in height steadily and tends towards the intermediate film conjugate height $h_{2}=0.8848$, eventually reaching a steady solution in a moving frame of reference, correlating to a travelling wave in a stationary frame of reference. Shuaib [23] also reports this type of wave profile with $\operatorname{Re}^{*}=0$. When inertial effects are considered (figure 8b); two wave fronts develop - a leading and a trailing front, giving a RUC-C combination. The trailing (left hand) front, although appearing to propagate in the negative direction, in the corresponding fixed reference (stationary frame) system, is moving upwards with a velocity slower than the reference one while the right hand side corresponds to an under-compressive front moving faster than the reference velocity. Both the leading and the trailing fronts are travelling up the plane in a stationary frame of reference. The height of the trailing front in figure $8 \mathrm{~b}$ is decreasing with time, and it is anticipated that this will ultimately tend towards the height of the left hand boundary. The height of the front on the right hand side has also decreased with time and is tending towards a compressive shock front, noting also the capillary ripples at its base. The results in figure 8 confirm uniqueness of solution with respect to figure 6. As time progresses the film profiles in figures $8 \mathrm{a}$ and $8 \mathrm{~b}$ tend to those in figures $6 \mathrm{a}$ and $6 \mathrm{c}$ respectively, corresponding to differences in the Reynolds numbers.

Figure 9, with boundary conditions $h_{l}=h_{2}, h_{r}=h_{3}$, illustrates the smoothing effect of inertia from an initial steep reverse compressive front for $\mathrm{Re}^{*}=0$ with a profile that develops into a steady solution in a moving frame of reference, physically representing a standing wave travelling up the plane. With the inclusion of inertia the profile evolves with time, becoming smoother and in figure $9 \mathrm{~b}$ can be seen moving in the negative $x$ direction at a non-dimensional speed of 0.61 . This is slower than the non-dimensional reference velocity of -1 , at which the entire system is moving, and so the net movement of this wave structure is up the plane, in the direction of the applied surface shear. Similar profiles are observed in figure 10, where the boundary conditions match but the initial profile contains an addition tier $h_{m}=h_{1}$ in the interior of the domain. Once again, the $\mathrm{Re}^{*}=0$ profile represents a travelling wave solution comparable to figure 9 a. When increasing the Reynolds number (figure 10b), we obtain a smoothed C-RUC profile that is evolving with time as in figure $9 \mathrm{~b}$.

Figure 11 illustrates the development of two-tier initial profiles with $h_{l}=h_{1}$ and $h_{r}=h_{3}$, while figure 12 contains three-tier initial profiles with the same boundary conditions and an interior tier at $h_{m}=h_{2}$. Again, similarity is observed between different profiles provided the boundary conditions are consistent, as illustrated with figure 11 and 12 , highlighting uniqueness of the solutions obtained. At $\operatorname{Re}^{*}=0$, we observe a central tier developing at $h=0.4374$, to the left of the main standing wave. This is not a solution of the cubic equation (3.7) which would be consistent with the flux at the inlet and outlet. The profiles in figures 11a and 12a are similar to a solution obtained by Levy and Shearer, figure 4.4 [12], who reported a large downward facing travelling wave followed by a smaller upward facing travelling wave. Although both waves are moving upwards, the speed of the larger wave is greater than that of the smaller one and the distance between them increases, leaving the interior film depth at $h=0.4374$. Increasing the Reynolds number, 
in both the two-tier and three-tier initial profile cases, has the effect of eliminating this feature, as well as smoothing the solution. Both inertial profiles $11 \mathrm{~b}$ and $12 \mathrm{~b}$ are of the same structure and the presence of the tier at $h_{m}=h_{2}$ in the interior of the three-tier initial profile does not affect the structure formed as time increases, once again indicating uniqueness of the solutions.

A typical rarefaction wave structure (figure 13) was also observed, such as in figure 5b of [23], with boundary conditions $h_{l}=h_{1}, h_{r}=h_{2}$. Increasing the Reynolds number had a minimal effect in this type of film profile, varying the speed of evolution, but with little change to the overall structure of the profile. When investigating the three-tier profile with boundary conditions equivalent to the rarefaction wave profile, we observe a decaying UC-Rw film profile as in figure 14. It is anticipated that this is a transient process tending towards the rarefaction wave over an extended period of time. The most significant difference between the non-inertial and inertial profiles in figures $14 \mathrm{a}$ and $14 \mathrm{~b}$ is the smoothing of the wave structure, as is expected with increasing Reynolds number.

The cases exhibited in figures 6 to 14 are representative of the types of film profile obtained at most angles of inclination investigated. However, on increasing the slope inclination to $\theta=-80$, the evolution of the film profile at $\operatorname{Re}^{*}=0$ reveals a wave feature not evident at $\theta=-45$. At $0 \leq \operatorname{Re}^{*} \ll 1$, the formation of an interior tier at a height of 1.9 is observed as in figure 15a. When increasing the film Reynolds number, the profile becomes identical in structure to the case at $\theta=-45$ with equivalent boundary conditions (figure 8 ). This feature is observed in cases where $h_{r}=h_{1}$ irrespective of whether the initial profile is of a two-tier or three-tier configuration and is classified as a compressive-undercompressive structure. The compressive wave is moving to the left of the domain at a slower speed than the reference velocity and therefore physically represents a wave structure moving in the positive $x$-direction, up the plane. The undercompressive wave is also moving up the plane, but at a faster rate, and so the tier at $h=1.9$ will become increasingly dominant throughout the domain. The inclusion of a small film Reynolds number is enough to eliminate this feature, as in figure 15b, and increasing the Reynolds number further (figure 15c) illustrates the uniqueness of the solution in comparison to figure $6 \mathrm{c}$.

\subsection{Formation of new fronts}

A new feature observed with the inclusion of inertia is the formation of an evolving region that is not a conjugate height in table 1 . The heights of the fronts depend on the boundary conditions of the initial profiles. In order to verify that the effects observed are a physical feature, the initial conditions are modified to maintain the same boundary conditions, but with modified conditions in the interior of the domain.

A profile initiated by $h_{l}=h_{3}$ (figures 16-19) produces an undercompressive front at $\operatorname{Re}^{*}=0$, also reported by Levy and Shearer [12]. We observe commonality of solution exhibited by changes in initial profile with equivalent boundary conditions developing into identically shaped wave structures. This is evident in figures $16 \mathrm{a}$ and the equivalent three-tier initial profile solution 17a, where $h_{m}=h_{1}, h_{r}=h_{2}$, as well as with figures 18a and 19a, where $h_{m}=h_{2}, h_{r}=h_{1}$. When increasing the Reynolds number, we observe the formation of a secondary undercompressive front - this is a new feature not reported in any Stokes flow study. The height of the intermediate state depends on the boundary conditions, so different initial profiles with identical boundary conditions form intermediate fronts of the same height. In figure 16b, the secondary front has an approximate film height of 1.5 - higher than the intermediate film height $h_{2}=0.8848$ associated with an slope inclination of $\theta=-45$. As in the previous section, the features produced from two-tier initial profiles can be replicated by allowing three-tier initial profiles with the respective boundary conditions to develop, suggesting that these are unique solutions given the boundary conditions in figures 16 and 17 . The film profiles in figure 18a and 19a with boundary conditions $h_{l}=h_{3}, h_{r}=h_{1}$, both develop into identically structured undercompressive waves with both the two-tier and the three-tier initial profile configurations. With the inclusion of inertia, as can be seen in figures $18 \mathrm{~b}$ and $19 \mathrm{~b}$, there is development of a secondary undercompressive front with an approximate film height of 1 , marginally lower than the intermediate film height.

\section{Summary and conclusions}

Thin film flow models are extensively used in modelling flows in industrial contexts, typically those associated with Stokes flow $(\mathrm{Re}=0)$ or low Reynolds number flows, to identify characteristic film flow behaviour and potential wave structures. The development of newer industrial technologies may require the film flow to be 
associated with an increased Reynolds number. This study provides an extended formulation for thin film flow corresponding to moderate Reynolds number associated with highly sheared flows. The paper evaluates the effect of increasing Reynolds number on existing film solutions, potential new wave structures and the influence of capillary ripples.

Model formulation uses a depth-averaged approach leading to a formulation similar to a Shkadov model approach but, importantly, incorporating surface tension effects which, together with a balance of gravity and surface shear, allows combinations of two or more uniform steady flow solutions. Characteristic wave solutions for increasing Reynolds number are sought involving shock structures linking these conjugate states. Numerical solutions are obtained using a finite-difference scheme with suitable upwinding applied. The formation of capillary ripples at the base of the main shock can happen, as reported in previous literature, and potential exaggeration of the amplitude of these ripples is a known problem of the Shkadov integral boundary layer model, leading to numerical singularities above a certain threshold Reynolds number. However this is not a critical issue in our work as the inclusion of surface shear and surface tension is shown to decrease the amplitude of these capillary ripples, whilst increasing their wavelength. The profiles exhibit uniqueness of solution, in the sense that initial profiles of identical boundary conditions develop into identically structured waves, providing credible evidence that these waves structures are physically representative of the model. Thus, the revised Shkadov model proposed in this study, together with the finite difference numerical implementation, has been able to obtain solutions for an extended range of Reynolds numbers for which the thin-film approximation is valid.

For modelling Stokes flow we were able to reproduce profiles obtained in previous literature. The Reynolds number was then increased to allow the initial profiles such as those illustrated in figures 4 and 5 to develop under the influence of inertia. For some initial profiles the inclusion of inertia had a marginal impact on the film profile. In other cases however, a significant change developed from the non-inertial profile. When comparing figures $17 \mathrm{a}$ and $17 \mathrm{~b}$, for example, both evolved from the same conjugate states selected from table 1. Developed profiles involved either modification and smoothing as the Reynolds number was increased, or the formation of fronts at intermediate (non-conjugate) heights. These heights were consistent throughout, with three-tier initial profiles producing similar wave structures to their two-tier counterparts when equivalent boundary conditions were applied.

In addition, we were also able to produce wave profiles present only at larger angles of slope inclination and demonstrate that changing the Reynolds number has a significant effect. Figure 15 illustrates, for an inclination angle of $\theta=-80$, the formation of a tier that is damped and then eliminated with the inclusion of a small film Reynolds number to the point where it is identical in structure to profiles obtained at $\theta=-45$.

Acknowledgements This work was carried out at the University Technology Centre in Gas Turbine Transmission Systems at the University of Nottingham with financial support from Rolls-Royce plc, Aerospace Group. The views expressed in this paper are those of the authors and not necessarily those of Rolls-Royce plc, Aerospace Group. We are also grateful for access to the University of Nottingham High Performance Computing Facility. 


\section{References}

[1] E.S. Benilov, Depth-averaged model for hydraulic jumps on an inclined plate, Physical Review E 89(5), 053-013, (2014).

[2] D.J. Benney, Long waves on liquid films, Journal of Mathematics and Physics 45(1), 150-155 (1966).

[3] A.L. Bertozzi, A. Münch and M. Shearer, Undercompressive shocks in thin film flows, Physica D 134(4), 431-464 (1999).

[4] H.-C. Chang, Wave evolution on a falling film, Annual Review of Fluid Mechanics 26(1), 103-136 (1994).

[5] H.-C. Chang, E.A. Demekhin and S.S. Saprikin, Noise-driven wave transitions on a vertically falling film, Journal of Fluid Mechanics 462, 255-283 (2002).

[6] M. Cheng and H.-C. Chang, Competition between subharmonic and sideband secondary instabilities on a falling film, Physics of Fluids 7(1), 34-54 (1995).

[7] G.F. Dietze, A. Leefken and R. Kneer, Investigation of the backflow phenomenon in falling liquid films, Journal of Fluid Mechanics 595, 435-459 (2008).

[8] B. Gjevik, Occurrence of finite-amplitude surface waves on falling liquid films, Physics of Fluids 13(8), 1918-1925 (1970).

[9] E.D. Kay, S. Hibberd and H. Power, Inertial effects at moderate Reynolds number in thin-film rimming flows driven by surface shear, Physics of Fluids 25(10), 102-108 (2013).

[10] N. Kofman, S. Mergui and C. Ruyer-Quil, Three-dimensional instabilities of quasi-solitary waves in a falling liquid film, Journal of Fluid Mechanics 757, 854-887 (2014).

[11] L. Kondic, Instabilities in gravity driven flow of thin fluid films, Siam Review 45(1), 95-115 (2003).

[12] R. Levy and M. Shearer, Kinetics and nucleation for driven thin film flow, Physica D 209(1), 145-163 (2005).

[13] N.A. Malamataris, M. Vlachogiannis and V. Bontozoglou, Solitary waves on inclined planes: flow structure and binary interactions, Physics of Fluids 14(3), 1082-1094 (2002).

[14] L.T. Nguyen and V. Balakotaiah, Modeling and experimental studies of wave evolution on free falling viscous films, Physics of Fluids 12(9), 2236-2256 (2000).

[15] P.J. Oliveira, F.T. Pinho and G.A. Pinto, Numerical simulation of non-linear elastic flows with a general collocated finite-volume method, Journal of Non-Newtonian Fluid Mechanics 79(1), 1-43 (1998).

[16] Th. Prokopiou, M. Cheng and H.-C. Chang, Long waves on inclined films at high Reynolds number, Journal of Fluid Mechanics 222, 665-691 (1991).

[17] C. Ruyer-Quil and P. Manneville, Modelling film flows down inclined planes, The European Physics Journal B 6(2), 277-292 (1998).

[18] C. Ruyer-Quil and P. Manneville, Improved modelling film flows down inclined planes, The European Physics Journal B 15(2), 357-369 (2000).

[19] A. Samanta, Shear-imposed falling film, Journal of Fluid Mechanics 753, 131-149 (2014).

[20] A. Samanta, C. Ruyer-Quil and B. Goyeau, A falling film down a slippery inclined plane, Journal of Fluid Mechanics 684, 353-383 (2011).

[21] B. Scheid, C. Ruyer-Quil, U. Thiele, O.A. Kabov, J.C. Legros and P. Colinet, Validity domain of the Benney equation including the Marangoni effect for closed and open flows, Journal of Fluid Mechanics 527, 303-335 (2005). 
[22] V.Ya. Shkadov, Wave flow regimes of a thin layer of viscous fluid subject to gravity, Fluid Dynamics, 2(1), 29-34 (1967).

[23] N.H. Shuaib, H. Power, S. Hibberd and K. Simmons, A numerical study of wave structures developed on the free surface of a film flowing on inclined planes and subjected to surface shear, International Journal for Numerical Methods in Engineering 68(7), 755-789 (2006).

[24] D.A. Tushkanov and V.Ya.Shkadov, Nonlinear waves in a liquid film on a near-horizontal surface, Fluid Dynamics 41(3), 330-342 (2006).

[25] L.-Q. Yu, F.K. Wasden, A.E. Dukler and V. Balakotaiah, Nonlinear evolution of waves on falling films at high Reynolds numbers, Physics of Fluids 7(8), 1886-1902 (1995). 


\section{$7 \quad$ Figures and tables}

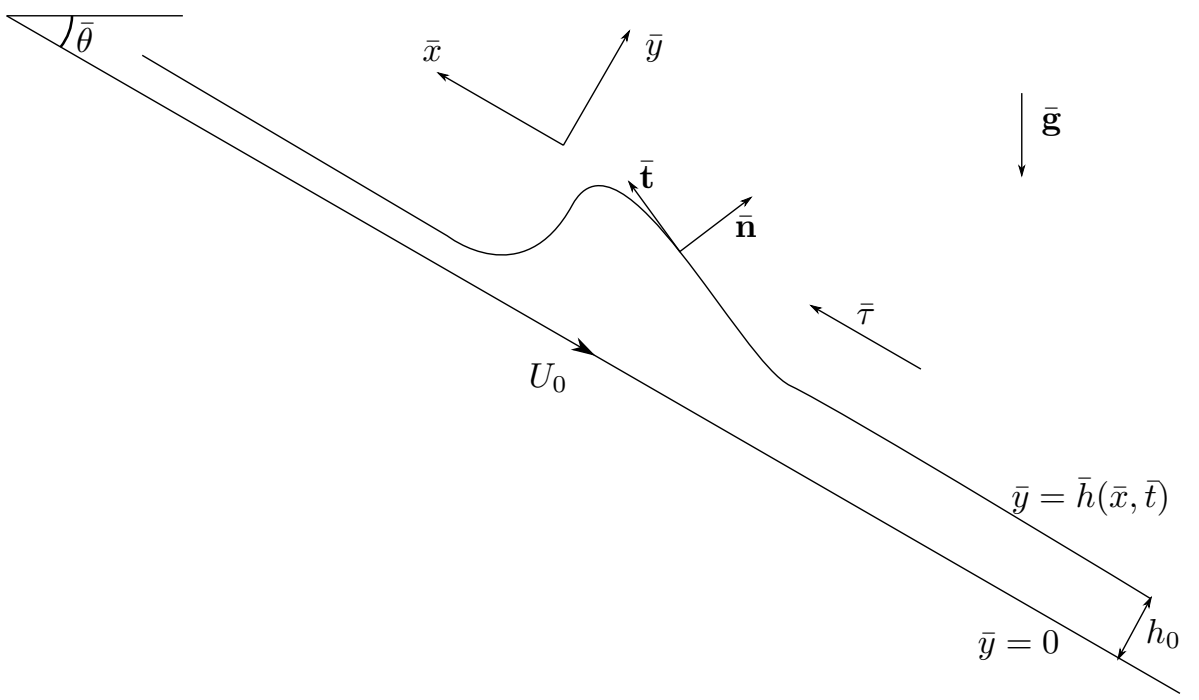

Figure 1: Plane geometry in dimensional coordinate system

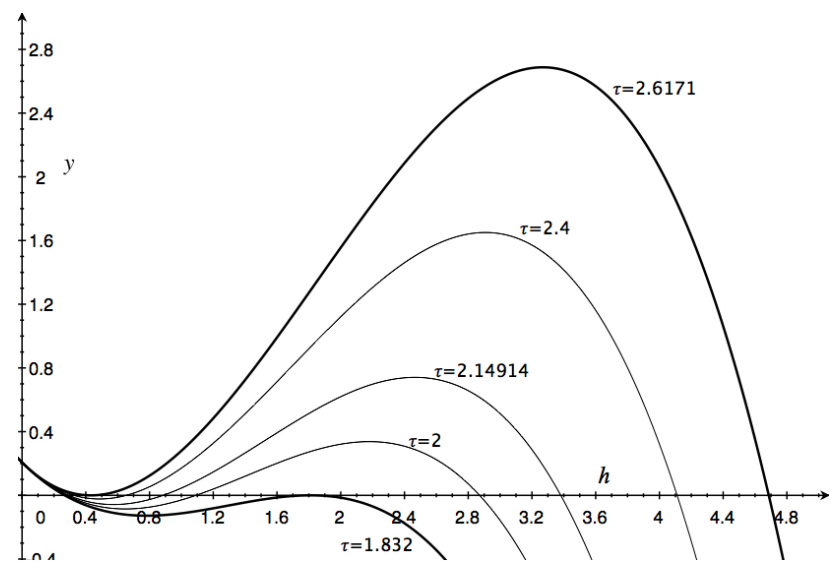

Figure 2: Cubic polynomial $y=\frac{\lambda \sin \theta}{3} h^{3}+\frac{\tau}{2} h^{2}-h-q$ plotted for increasing values of $\tau$. Parameters: $\lambda=1$, $\theta=-45, q=-0.2068$.

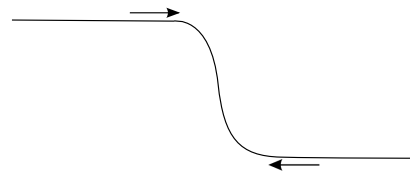

(a) Compressive (C)

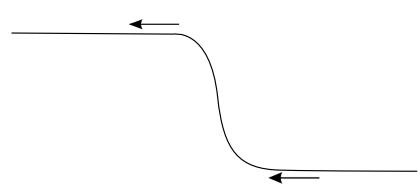

(b) Undercompressive (UC)

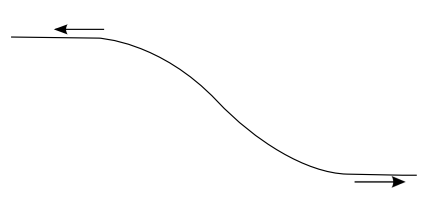

(c) Rarefaction wave $(\mathrm{Rw})$

Figure 3: Direction of propagation of perturbations in a moving frame of reference. 


\begin{tabular}{|c|c|c|c|c|}
\hline$\tau$ & $\theta$ & $h_{1}$ & $h_{2}$ & $h_{3}$ \\
\hline 2 & -45 & 0.2801 & 1.0910 & 2.8716 \\
\hline 2.14914 & -45 & 0.2933 & 0.8848 & 3.3809 \\
\hline 2.4 & -45 & 0.3266 & 0.6535 & 4.1111 \\
\hline 2.14914 & -80 & 0.2822 & 1.2878 & 1.6975 \\
\hline
\end{tabular}

Table 1: Conjugate values of $h$ for $q=-0.2068$ and $\lambda=1$.

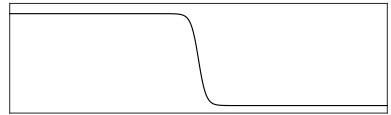

(a) $h_{l}>h_{r}$

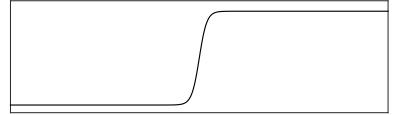

(b) $h_{l}<h_{r}$

Figure 4: Two-tier initial profiles

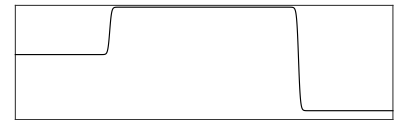

(a) $h_{m}>h_{l}>h_{r}$

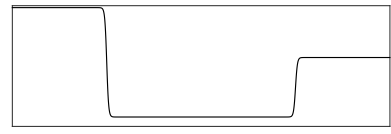

(d) $h_{l}>h_{r}>h_{m}$

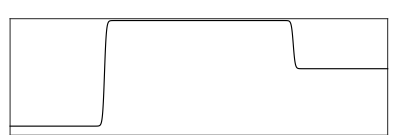

(b) $h_{m}>h_{r}>h_{l}$

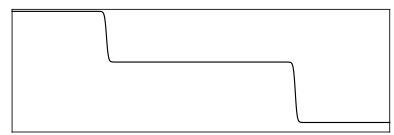

(e) $h_{l}>h_{m}>h_{r}$

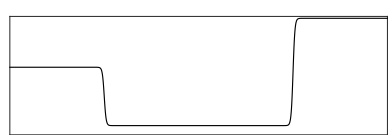

(c) $h_{r}>h_{l}>h_{m}$

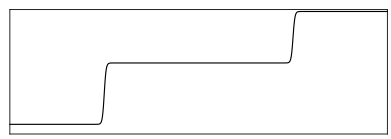

(f) $h_{l}<h_{m}<h_{m}$

Figure 5: Three-tier initial profiles 


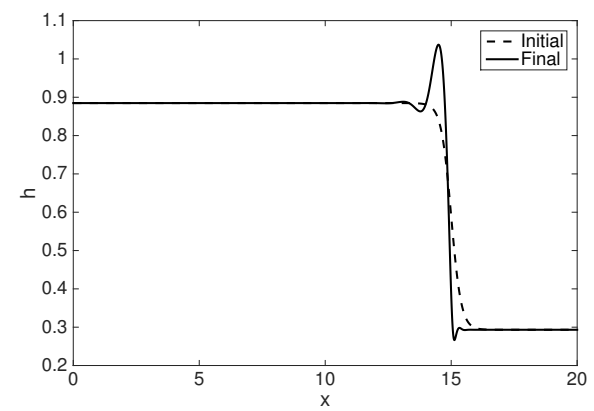

(a) $\operatorname{Re}^{*}=0$

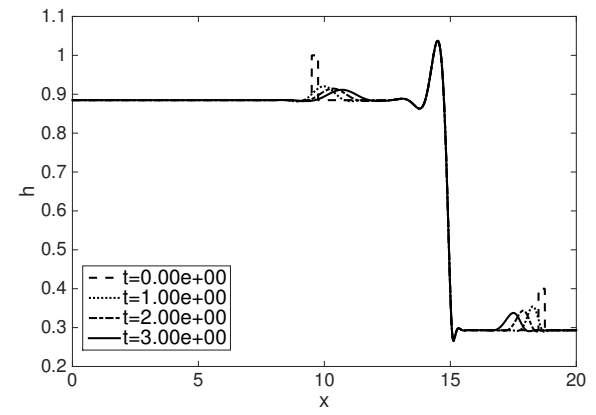

(b) $\operatorname{Re}^{*}=0$ with perturbations

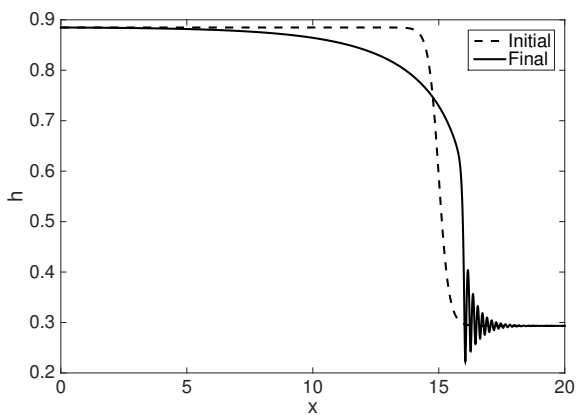

(c) $\operatorname{Re}^{*}=10$

Figure 6: Compressive. $\theta=-45, h_{l}=0.8848, h_{r}=0.2933$

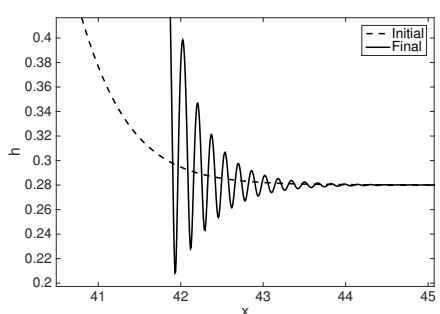

(a) $\tau=2$

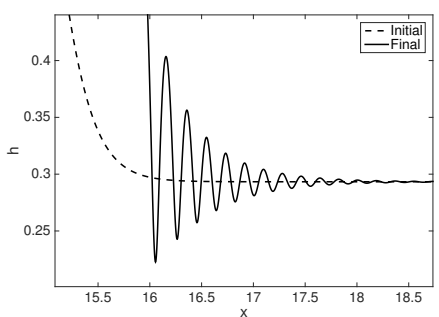

(b) $\tau=2.14914$ (figure 6c)

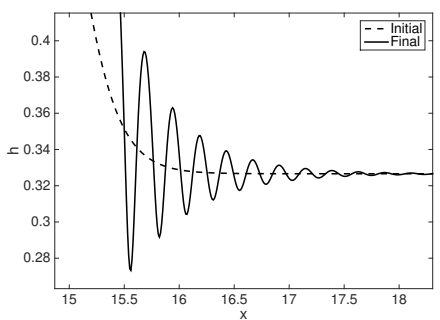

(c) $\tau=2.4$

Figure 7: Capillary waves for increasing values of $\tau$ at $\operatorname{Re}^{*}=10$.

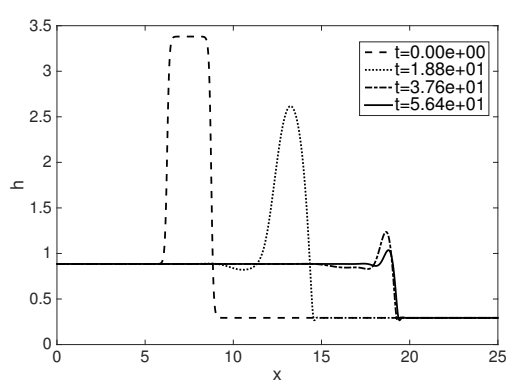

(a) $\operatorname{Re}^{*}=0$

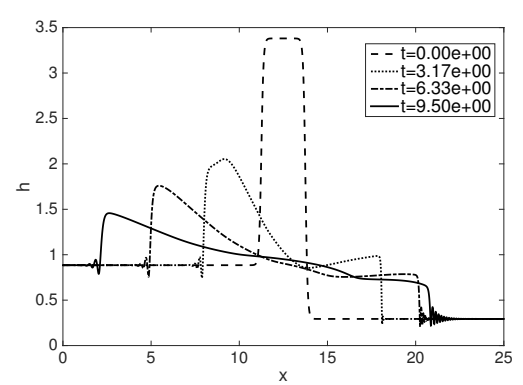

(b) $\operatorname{Re}^{*}=10$

Figure 8: (a) Compressive. (b) Reverse undercompressive - compressive. $\theta=-45, h_{l}=0.8848, h_{m}=3.3809$, $h_{r}=0.2933$ 


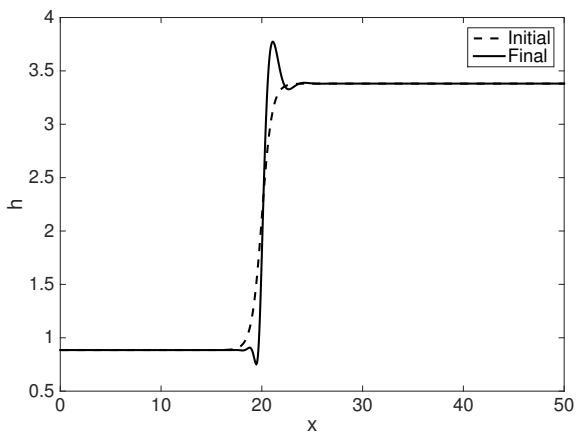

(a) $\operatorname{Re}^{*}=0$

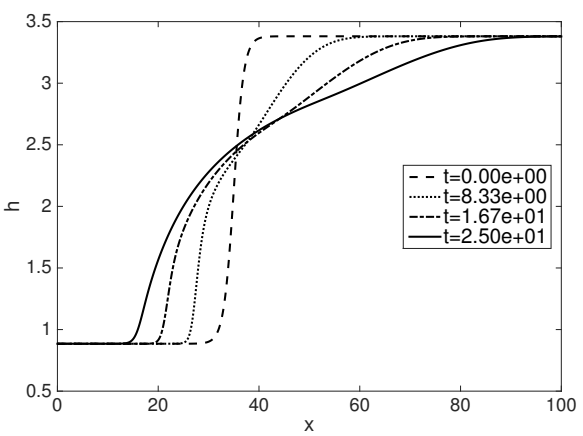

(b) $\mathrm{Re}^{*}=10$

Figure 9: Reverse compressive: $\theta=-45, h_{l}=0.8848, h_{r}=3.3809$

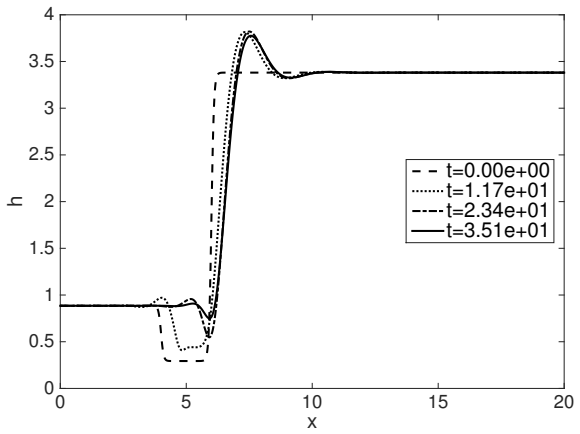

(a) $\operatorname{Re}^{*}=0$

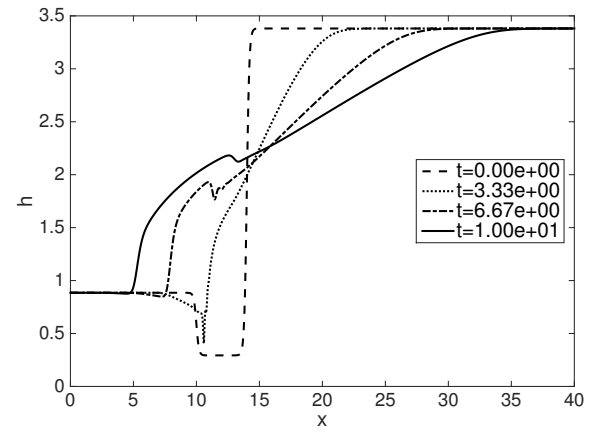

(b) $\operatorname{Re}^{*}=10$

Figure 10: (a) Compressive. (b) Compressive - reverse undercompressive. $\theta=-45, h_{l}=0.8848, h_{m}=$ $0.2933, h_{r}=3.3809$

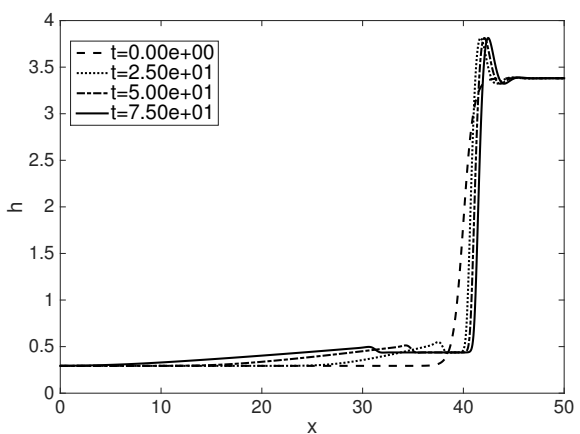

(a) $\operatorname{Re}^{*}=0$

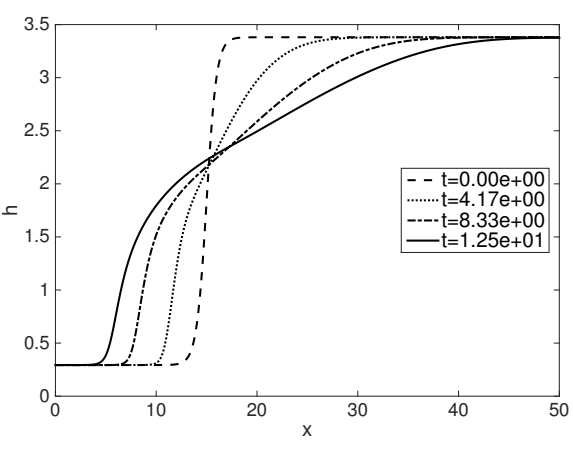

(b) $\operatorname{Re}^{*}=10$

Figure 11: Undercompressive - reverse undercompressive. $\theta=-45, h_{l}=0.2933, h_{r}=3.3809$ 


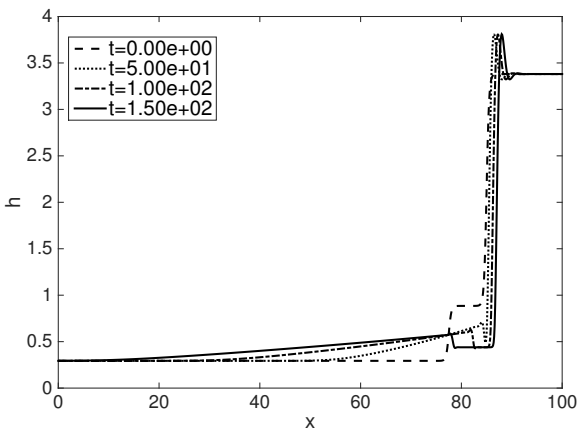

(a) $\operatorname{Re}^{*}=0$

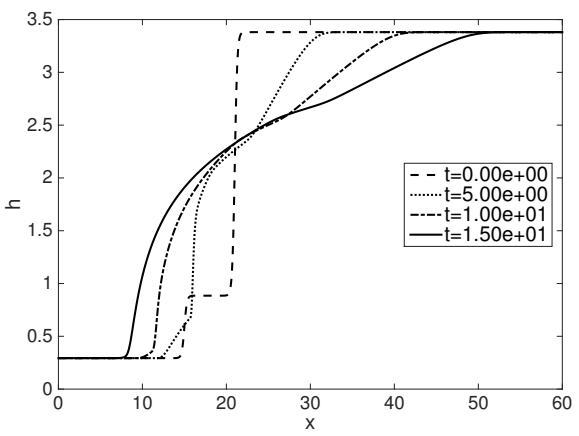

(b) $\mathrm{Re}^{*}=10$

Figure 12: Undercompressive - reverse undercompressive. $\theta=-45, h_{l}=0.2933, h_{m}=0.8848, h_{r}=3.3809$

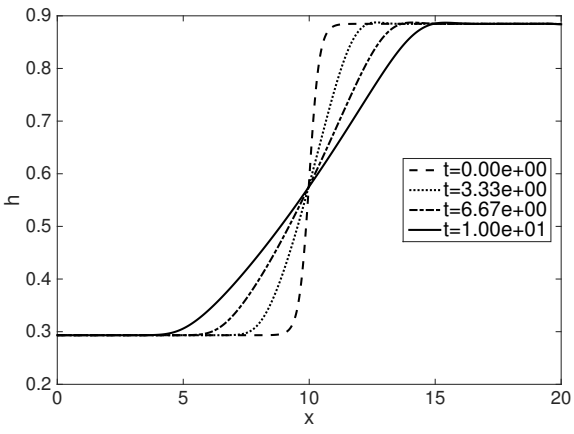

(a) $\operatorname{Re}^{*}=0$

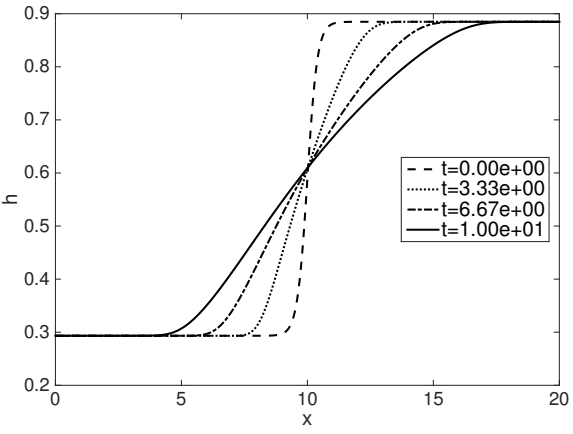

(b) $\mathrm{Re}^{*}=10$

Figure 13: Rarefaction wave. $\theta=-45, h_{l}=0.2933, h_{r}=0.8848$

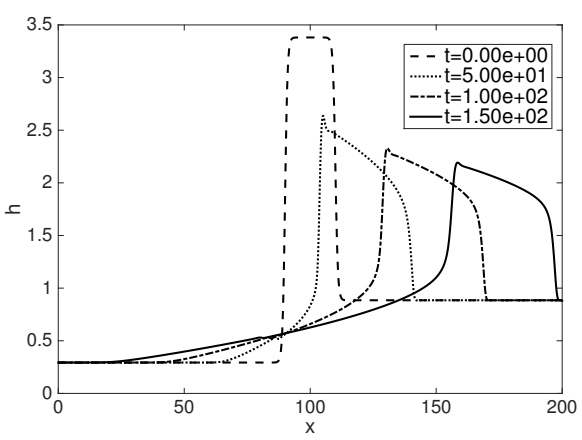

(a) $\operatorname{Re}^{*}=0$

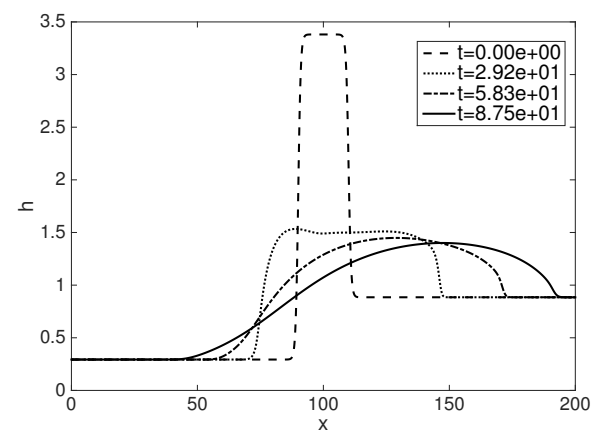

(b) $\mathrm{Re}^{*}=10$

Figure 14: Undercompressive - rarefaction wave. $\theta=-45, h_{l}=0.2933, h_{m}=3.3809, h_{r}=0.8848$ 


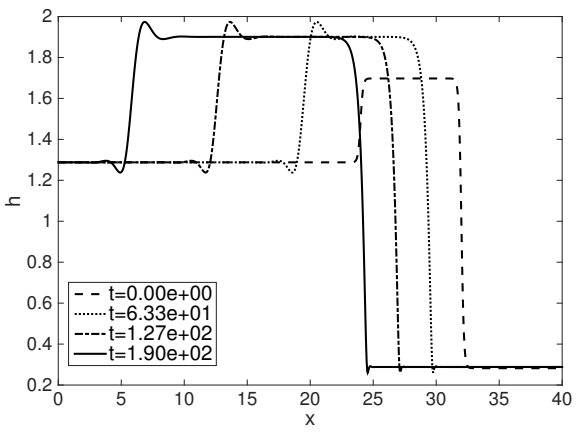

(a) $\operatorname{Re}^{*}=0$

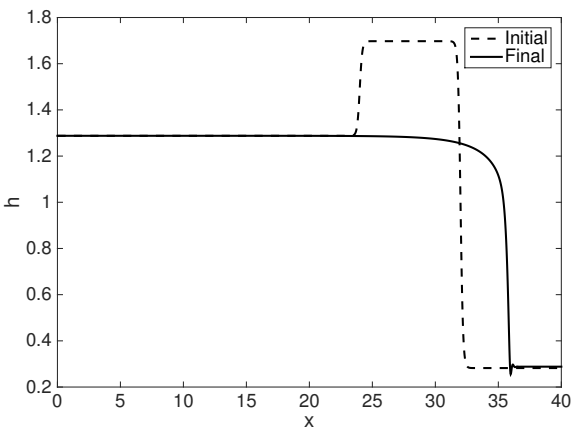

(b) $\operatorname{Re}^{*}=1$

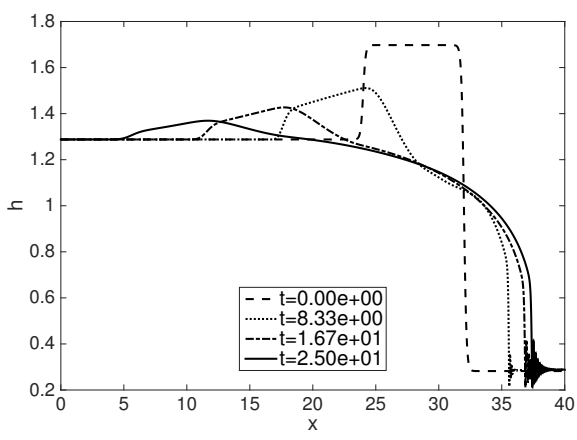

(c) $\operatorname{Re}^{*}=10$

Figure 15: (a) Compressive - undercompressive. (b) and (c) Compressive. $\theta=-80, h_{l}=1.2878, h_{m}=$ $1.6975, h_{r}=0.2822$

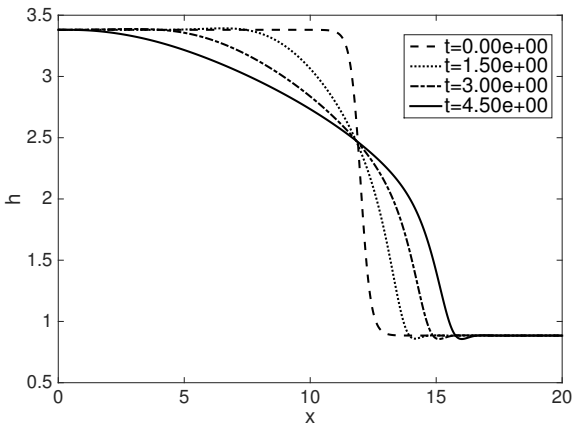

(a) $\operatorname{Re}^{*}=0$

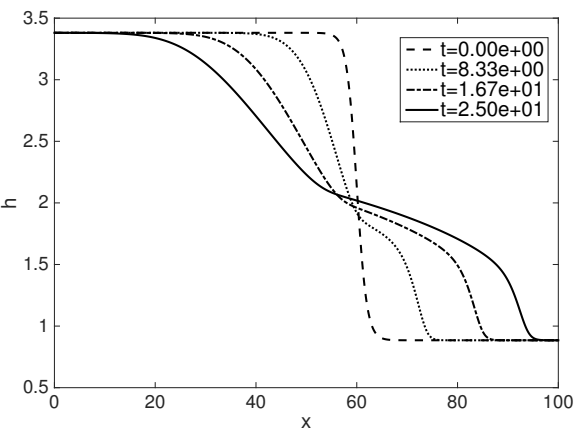

(b) $\operatorname{Re}^{*}=10$

Figure 16: (a) Undercompressive. (b) Undercompressive - undercompressive. $\theta=-45, h_{l}=3.3809$, $h_{r}=0.8848$ 


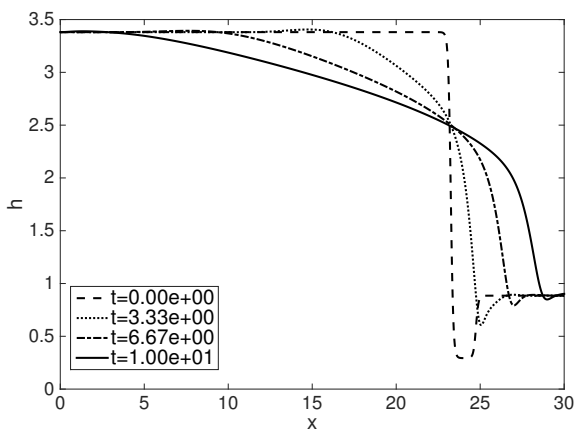

(a) $\operatorname{Re}^{*}=0$

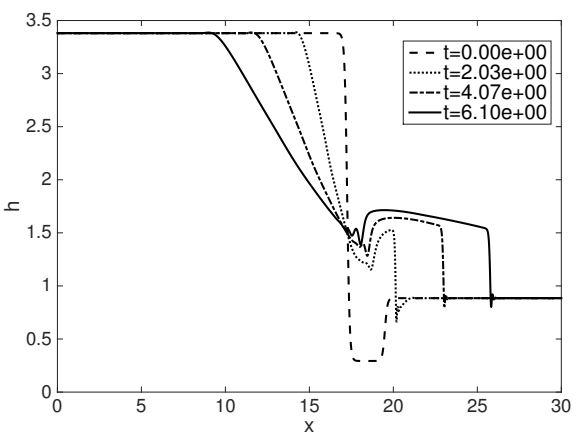

(b) $\operatorname{Re}^{*}=10$

Figure 17: (a) Undercompressive. (b) Undercompressive - undercompressive. $\theta=-45, h_{l}=3.3809$, $h_{m}=0.2933, h_{r}=0.8848$

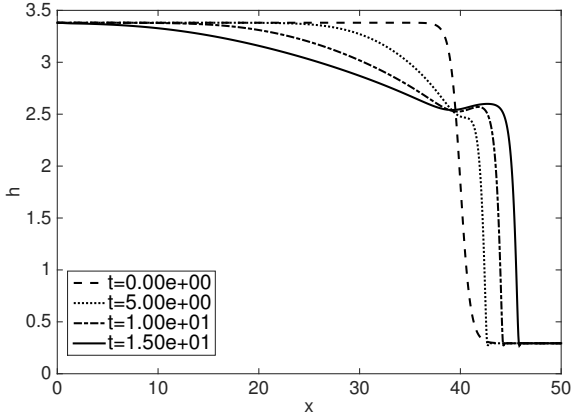

(a) $\operatorname{Re}^{*}=0$

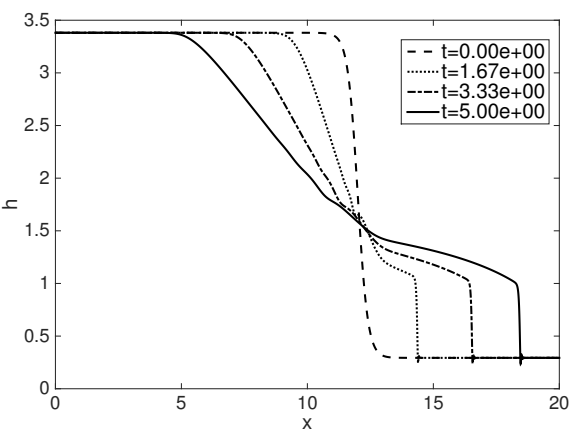

(b) $\operatorname{Re}^{*}=10$

Figure 18: (a) Undercompressive. (b) Undercompressive - undercompressive. $\theta=-45, h_{l}=3.3809$, $h_{r}=0.2933$

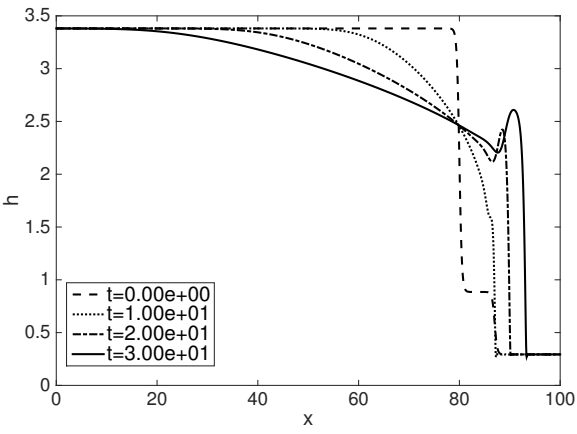

(a) $\operatorname{Re}^{*}=0$

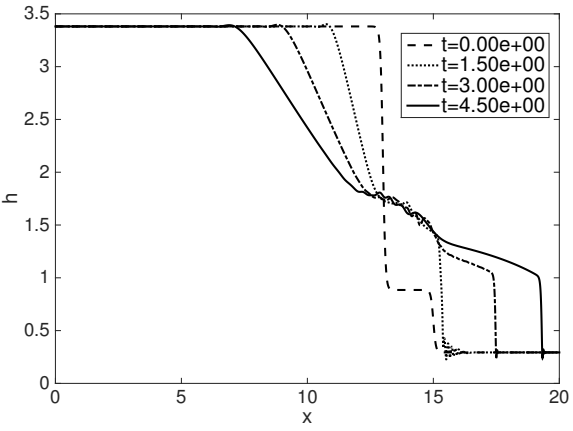

(b) $\operatorname{Re}^{*}=10$

Figure 19: (a) Undercompressive. (b) Undercompressive - undercompressive. $\theta=-45, h_{l}=3.3809$, $h_{m}=0.8848, h_{r}=0.2933$ 